\title{
Immune Checkpoint Inhibitors: Response Assessment and Immune-related Adverse Events
}

\author{
Section of NM and PET/CT Imaging Services, Department of Radiology, The Aga Khan University Hospital, Karachi, Pakistan
}

Maseeh uz Zaman and Nosheen Fatima

One of the most important discoveries of current era is that the evasion of immune system is the hallmark of cancer development. ${ }^{1}$ The immune system is able to recognise antigens derived from cancer cells and; therefore, distinguishes them from their normal counterparts to generate a tumor-specific T-cell mediated immune response. Two inhibitory proteins (inhibitory checkpoints) have been identified leading to immune evasion, which include cytotoxic T-lymphocyte associated protein-4 (CTLA-4) and programmed cell death protein-1 (PD-1) and its ligand (PD-L1). ${ }^{2}$ Based on these discoveries, various strategies have been devised to enhance cancer specific immune response. These include vaccines (like BCG for superficial urinary bladder cancer) ${ }^{3}$, cytokines, adaptive CAR-T cell (chimeric antigen receptor $\mathrm{T}$-cell), and immune checkpoint inhibitors (ICls). ${ }^{4}$ Among these, ICls are very much on therapeutic frontier in the current decade.

Two types of ICls have been introduced, which include Anti-CTLA-4 (like ipilimumab) and Anti-PD-1/PD-L1 (like nivolumab and pembrolizumab). ${ }^{5}$ These ICls have shown significant survival benefit in broad range of cancers like melanoma, head and neck cancers, renal cell and transitional cell cancer, non-small cell lung cancer and refractory lymphomas. ${ }^{6}$ Due to exemplary promising effects by these agents, Food and Drug Administration (FDA) approved these agents for clinical use in 2011. ICls reactivate the immune system and unleash the T-cells to recognise and kill cancer cells. ${ }^{2}$ This is demonstrated by heavy T-lymphocytes infiltration in tumor cells around the necrotic foci. ${ }^{7}$ These T-cells have affinity for 18-flourine-deoxyglucose (18-FDG) as shown by Takayoshi in 20028; and this paved the path of 18-FDG positron emission tomography and computerised tomography (PET/CT) for imaging response evaluation to ICls. Like other therapeutic options used in oncology, the response to $\mathrm{ICls}$ can be evaluated both on morphological and metabolic criteria. However, it is

Correspondence to: Professor Maseeh uz Zaman, Section of NM \& PET/CT Imaging Services, Department of Radiology,

The Aga Khan University Hospital, Stadium Road, Karachi,

Pakistan

E-mail:maseeh.uzzaman@aku.edu

Received: July 02, 2019; Revised: August 22, 2019;

Accepted: August 22, 2019 important to understand that response to ICls is different from chemotherapeutic and molecularly targeted agents, as it appears quite early; but delayed appearance is not uncommon. 9 So far various response patterns have been observed for ICls, which include profound and durable tumour shrinkage, pseudo-progression and hyper-progression. ${ }^{10}$ It is also important to get acclimatised about novel immune-related adverse effects (irAEs), which are also different from chemotherapeutic or molecularly targeted agents.10 Pseudo-progression is defined as apparent disease progression with initial increase in tumour size with or without appearance of new lesions, but without deterioration of clinical status. ${ }^{11}$ It is commonly observed in early phase of treatment and usually followed by reduction or disappearance of tumour or a stable disease pattern. This paradox in imaging pattern and clinical status is caused by recruitment of activated T-cells, which recognise and kill tumor cells (immune flare). ${ }^{10}$ This immune flare is responsible for increase in tumour size and metabolism, which can be assessed on anatomical (computerised CT, MRI or ultrasound) and functional (18-FDG based PET) imaging.10 Pseudoprogression is seen in about $15 \%$ of patients with melanoma and those on anti-CTLA4 (ipilimumab) and less common $(5 \%)$ in other tumours and patients treated with anti-PD1/anti-PD-L1 (nivolumab). ${ }^{10}$ Based on the results of initial trials, recent recommendation is to continue treatment with $\mathrm{ICl}$ followed by a follow-up imaging at least after 4 weeks, but not later than 8 weeks. ${ }^{10}$ Pseudo-progression is a challenging situation, both for reporting physicians and oncologists. So far, no valid biochemical or imaging marker is available to confirm pseudo-progression. ${ }^{1}$ Tumour biopsy is considered the gold standard, which is usually not practical in many cases. Some initial studies have shown that the higher circulating tumour DNA at baseline reduces the probability of pseudo-progression in due course of treatment. ${ }^{1}$ Hyper-progression is defined as increase in tumour growth after the initiation of treatment with ICls by a factor of two. ${ }^{10}$ It is not clearly known whether it is caused by tumour behaviour or ICl-induced acceleration because most of the initial trials did not have control arms. ${ }^{1}$ In contrary to pseudo-progression, hyperprogression is associated with symptomatic deterioration, more commonly (9-29\%), occurs in all types of tumours 
with a lower incidence of appearance of new lesions. ${ }^{1}$ According to recent recommendations, if hyperprogression is suspected, $\mathrm{ICl}$ should be discontinued, as it is associated with worst prognosis. 1

Like chemotherapy or molecularly targeted agents, response evaluation criteria in solid tumor (RECIST) is also used for ICls response evaluation.9 In 2009, Wolchok et al., used RECIST 1.1 (unidimensional) and World Health Organization (WHO) criteria (2-dimentional) on a small group of patients with melanoma treated with ipilimumab. ${ }^{7}$ They observed that using either size-based criteria, resulted in premature discontinuation of potentially effective therapy in a small group of patients. This encouraged them to design first immune-related response criteria (irRC). They found that $10 \%$ of patients being treated with $\mathrm{ICl}$ moved from progressive disease (PD) as per WHO criteria to partial response (irPR) or stable disease (irSD) as per irRC due to compatible overall survival (OS). ${ }^{7}$ This was the point when pseudoprogression was recognised as an established response to ICls. This was followed by publication of new immunerelated RECIST (irRECIST) criteria by Nishino et al. in $2013^{12}$ and another in 2017 by RECIST group as iRECIST. 13 In irRC and irRECIST criteria, new lesion(s) is incorporated in the sum of measurements while iRECIST considers it as unconfirmed progressive disease (iUPD) which becomes confirmed progressive disease (iCPD) on follow-up.

There are two well established metabolic criteria which are used in PET/CT imaging. ${ }^{10}$ First was produced by European Organization of Research and Treatment of Cancer (EORTC) in 1999. It uses maximum standardised uptake value (SUVmax) as semi-quantitative parameter (maximum single pixel-based value). ${ }^{10}$ SUVmax is the most commonly used parameter worldwide due to convenience in using it despite issue of reproducibility. EORTC criteria was used for ICI response evaluation in 2015. Second criteria is PET-response evaluation criteria in solid tumor (PERCIST), published by Richard Wahl in 2009.14 PERCIST recommends to use SULpeak (SUV measured for body mass index over a $1 \mathrm{~cm}$ region of interest), which is more reproducible than SUVmax. In 2018, PERCIST was used for response evaluation for patients having ICls. In 2017, Cho et al., applied irRC, RECIST1.1, PERCIST, and EORTC criteria individually and a newly designed criteria (PECRIT: PET/CT criteria for early prediction of response to $\mathrm{ICl}$ ) upon a cohort of 20 melanoma patients. ${ }^{15}$ They found that diagnostic accuracy of PECRIT was $95 \%$, which was significantly higher than other morphological or metabolic criteria. PECRIT also categorised the patients into clinical benefit group (CB) for patients with complete metabolic response (CMR), partial metabolic response (PMR) or stable metabolic disease (SMD) with SULpeak $>15.5 \%$. While patients with PD or SMD with SULpeak $<15.5 \%$ are included in no clinical benefit (NCB) group. 15 In 2018,
Heidelberg group used a modified PERCIST criteria (PERCIMT: PET response evaluation criteria for immunotherapy) upon a cohort of 41 melanoma patients treated with ipilimumab. ${ }^{16}$ Like PECRIT criteria, they also categorised patients into clinical benefit (CMR, $P M R, S D)$ and no clinical benefit (PD) groups. However, they have emphasised upon using size and number of new lesions to diagnose PD. 16

It is also important for reporting physicians and treating oncologists to be cognizant of novel immune-related adverse effects (irAEs) as these are different from chemotherapy and molecularly targeted agents. ${ }^{10}$ irAEs can affect any organ as these are attributed to autoimmunity and T-cell infiltration. 18-FDG PET/CT plays an important role in diagnosing irAES due to their characteristic appearance on interim scan and resolution after steroid therapy on follow-up scan. ${ }^{9}$ Patient could have skin rash of variable severity or diarrhea due to colitis, which is more common with ipilimumab. Colitis is presented as diffuse 18-FDG uptake over large bowel in interim scan, which has to be differentiated from metformin (oral hypoglycemic agent) induced by correlating with symptoms and drug history. $10 \mathrm{ICl}$-induced pneumonitis is more common with nivolumab; and usual presentation is dyspnea with enhanced 18-FDG uptake over both lungs on interim scan and resolution after steroid treatment on followup. 10 Sarcoid-like reaction is more common with pembrolizumab and appears as intense 18-FDG uptake on mediastinal nodes with famous "Lambda Sign". $10 \mathrm{ICls}$ could also involve pituitary-thyroid-adrenal axis with thyroiditis being the most common presentation. 10 Like pseudo-progression, irAEs are also considered as predictors of good response to ICls.

18-FDG PET/CT is considered more sensitive and informative than contrast enhanced CT (CECT) in assessing response to ICls. ${ }^{9}$ Each PET/CT report should have information about type of $\mathrm{ICl}$, number of cycles and date of last $\mathrm{ICl}$ administered. Report should also mention clinical symptoms related to irAEs and presence or absence of concomitant presentation on interim and follow-up scans. Report must also have a comment about timing of follow-up scan, which is usually advised after 2-3 cycles or after 8-9 weeks from current study. It is important to realise that overall clinical status of the patient must be communicated to reporting physician by referring oncologist to distinguish pseudoprogression or hyper-progression with a high level of confidence. 10

Currently, we have been living in the era of ICls which have shown significant survival benefit in various cancers. ICls have patterns of response and progression, which are usually different from chemotherapy and molecularly targeted agents. Pseudo-progression is an exception and not a rule, which occurs in $<10 \%$ cases 
and failure to recognise it could result in premature discontinuation of an effective treatment. This has also led to the development of new immune-related metabolic and morphological criteria for response evaluation. Hyper-progression is more common than pseudoprogression; and is defined by increase in tumour burden by a factor of two, which predicts worst prognosis. Hybrid imaging using 18-FDG PET/CT is a better modality than CECT for assessment of response to ICIs and early detection and follow-up of irAEs. Pseudoprogression and irAEs are considered predictor of good response to ICls. Till date, there is no sensitive and reliable biomarker available to precisely detect efficacy, pseudo-progression or hyper-progression in patients being treated with the ICls.

\section{REFERENCES}

1. Borcoman E, Nandikolla A, Long G, Goel S. Tourneau C. Patterns of response and progression to immunotherapy. 2018 ASCO Educational Book | asco.org/edbook (visited on 20.6.19).

2. Pardoll DM. The blockade of immune checkpoints in cancer immunotherapy. Nat Rev Cancer 2012; 12:252-64.

3. Hanahan D, Weinberg RA. Hallmarks of cancer: The next generation. Cell 2011; 144:646-74.

4. Okazaki T, Honjo T. PD-1 and PD-1 ligands: From discovery to clinical application. Int Immunol 2007; 19:813-24.

5. Ott PA, Hodi FS, Robert C. CTLA-4 and PD-1/PD-L1 blockade: New immunotherapeutic modalities with durable clinical benefit in melanoma patients. Clin Cancer Res 2013; 19:5300-9.

6. Ferte $\mathrm{C}$, Marabelle A. iRECIST: A clarification of tumour response assessment in the immunotherapy era. Eurp J Canc 2017; 77: 165-7.

7. Wolchok JD, Hoos A, O'Day S, Weber JS, Hamid O, Lebbé C, et al. Guidelines for the evaluation of immune therapy activity in solid tumors: Immune-related response criteria. Clin Cancer Res 2009; 15:7412-20.

8. Takayoshi I, Saga T, Mamede M, Kobayashi H, Higashi T, Nakamoto $\mathrm{Y}$, et al. Increased 18F-FDG uptake in a model of inflammation: concanavalin A-mediated lymphocyte activation. J Nucl Med 2002; 43:658-63.

9. Mizuki N, Nikhil R, Hatabu H, Hodi FS. Monitoring immunecheckpoint blockade: Response evaluation and biomarker development. Nat Rev Clin Oncol 2017; 14:655-68.

10. Nicolas A, Hicks RJ, Tourneau C, Lheureux S, Fanti S, Lopci E. FDG PET/CT for assessing tumour response to immunotherapy. Eur J Nucl Med Mol Imaging 2019; 46:238-50.

11. Wolchok JD, Hoos A, O'Day S, Weber JS, Hamid O, Lebbe C, et al. Guidelines for the evaluation of immune therapy activity in solid tumors: Immune-related response criteria. Clin Cancer Res 2009; 15:7412-20.

12. Nishino M, Giobbie HA, Gargano M, Suda M, Ramaiya NH, Hodi FS. Developing a common language for tumor response to immunotherapy: Immune-related response criteria using unidimensional measurements. Clin Cancer Res 2013; 19:3936-43.

13. Seymour L, Bogaerts J, Perrone A, Ford R, Schwartz LH, Mandrekar, S, et al. iRECIST: Guidelines for response criteria for use in trials testing immunotherapeutics. Lancet Oncol 2017; 18:143-52.

14. Wahl RL, Jacene $H$, Kasamon $Y$, Lodge MA. From RECIST to PERCIST: Evolving considerations for PET response criteria in solid tumors. J Nucl Med 2009; 50 (Suppl 1):122S-50S.

15. Cho SY, Lipson EJ, Im HJ, Rowe SP, Gonzalez EM, Blackford A, et al. Prediction of response to immune checkpoint inhibitor therapy using early-time-point (18)F-FDGPET/CTimaging in patients with advanced melanoma. J Nucl Med. 2017; 58:1421-28.

16. Anwar H, Sachpekidis C, Winkler J, Kopp-Schneider A, Haberkorn U, Hassel JC, et al. Absolute number of new lesions on (18)F-FDG PET/CT is more predictive of clinical response than SUV changes in metastatic melanoma patients receiving ipilimumab. Eur J Nucl Med Mol Imaging 2018; 45:376-83. 